\title{
Geodesic Knots in the Figure-Eight Knot Complement
}

Sally M. Miller

\section{CONTENTS}

1. Introduction

2. Preliminaries

3. Geodesics in the Figure-Eight Knot Complement

4. Closed Orbits in Suspension Flows

5. Conclusion

6. Geodesic Symmetry Orbit Representatives

Acknowledgements

References

Supported in part by an Australian Postgraduate Award from the Commonwealth Government.

\begin{abstract}
We address the problem of topologically characterising simple closed geodesics in the figure-eight knot complement. We develop ways of finding these geodesics up to isotopy in the manifold, and notice that many seem to have the lowest-volume complement amongst all curves in their homotopy class. However, we find that this is not a property of geodesics that holds in general. The question arises whether under additional conditions a geodesic knot has least-volume complement over all curves in its free homotopy class.

We also investigate the family of curves arising as closed orbits in the suspension flow on the figure-eight knot complement, many but not all of which are geodesic. We are led to conclude that geodesics of small tube radii may be difficult to distinguish topologically in their free homotopy class.
\end{abstract}

\section{INTRODUCTION}

By a hyperbolic three-manifold we mean a complete orientable finite volume three-dimensional Riemannian manifold, all of whose sectional curvatures are -1 . Every hyperbolic three-manifold contains one (and typically more than one) simple closed geodesic - see [Adams et al. 1999], for example - and such a simple closed geodesic is also called a geodesic knot. In Section 2 and in [Miller $\geq 2001$ ] we show that large classes of hyperbolic three-manifolds in fact contain infinitely many geodesic knots.

In this paper we consider the problem of topologically characterising these geodesic knots - that is, we look for necessary or sufficient topological conditions for a simple closed curve $\gamma$ in a hyperbolic three-manifold $M$ to be isotopic to a geodesic. These conditions could either be properties of the curve $\gamma$ in $M$, or of its complement, the drilled manifold $M-\gamma$, which is hyperbolic if $\gamma$ is a geodesic; see [Kojima 1988] and Section 2. Here we investigate this in the case where $M$ is the figure-eight 
knot complement. To do this, we develop methods of locating geodesics 'explicitly' in the manifold by identifying their isotopy class within a free homotopy class of closed curves.

Our investigations rely heavily on the computer program SnapPea by Jeff Weeks, and its extensions Snap and Tube by Oliver Goodman. In particular, these programs can drill closed curves from hyperbolic three-manifolds, and provide a range of invariants associated with the resulting manifold.

The paper is organised as follows. In Section 2 we collect various mathematical results that lie behind the work we carry out. In particular we show that the figure-eight knot complement has infinitely many geodesic knots, thus giving a useful source to investigate. We also briefly discuss the features of SnapPea, Snap and Tube that we use (see [Hodgson and Weeks 1994; Coulson et al. 2000] for more details). In Sections 3 and 4 we discuss various aspects of the set of geodesic knots in the figure-eight knot complement. In Section 3 we use the computer programs to help identify closed geodesics in the figure-eight knot complement, and to help investigate properties of their complements. Section 4 continues this investigation, but uses the structure of the figure-eight knot complement as a once-punctured torus bundle over the circle. In Section 5 we gather together conclusions and suggest questions arising from this work.

\section{PRELIMINARIES}

This section presents several results about geodesics in hyperbolic three-manifolds.

\section{Geodesics in Hyperbolic 3-Manifolds}

For $d$ a squarefree positive integer, let $\mathcal{O}_{d}$ be the ring of integers in the quadratic imaginary number field $\mathbb{Q}(\sqrt{-d})$. Recall that, if we define $\omega$ as $\frac{1}{2}(1+\sqrt{-d})$ if $d \equiv-1 \bmod 4$ and as $\sqrt{-d}$ otherwise, then $\mathcal{O}_{d}=$ $\mathbb{Z}[\omega]$ and $\{1, \omega\}$ is a basis for $\mathcal{O}_{d}$.

Our first result shows that many hyperbolic manifolds contain an abundance of geodesic knots.

Proposition 2.1. If $M=\mathbb{H}^{3} / \Gamma$ is a hyperbolic threeorbifold such that $\Gamma$ is a finite index subgroup of the Bianchi group $\Gamma_{d}=\operatorname{PSL}\left(2, \mathcal{O}_{d}\right)$ for some squarefree positive integer $d$, then $M$ contains infinitely many simple closed geodesics.
In the case that $M$ is an orbifold whose singular locus contains a one-dimensional connected subset $\Sigma$ of order 2 , then we will call closed any simple geodesic which occurs in $M$ as a geodesic arc with endpoints in $\Sigma$. For, upon lifting with respect to the corresponding order 2 symmetry, the geodesic is closed in the usual sense.

Proposition 2.1 encompasses the case of link complements $M=S^{3}-k$ for such $k$ as the figure-eight knot, the Whitehead link, and the Borromean rings. Its proof uses the following fact:

Lemma 2.2. Let $g, h \in \operatorname{PSL}(2, \mathbb{C})$ with $\operatorname{tr} g \neq \pm 2$. Then the complex distance $\rho+i \theta$ between axis $g$ and its translate $h$ axis $g=$ axis $h g h^{-1}$ is given by

$$
\cosh (\rho+i \theta)=\frac{\operatorname{tr}^{2} g-2 \operatorname{tr}[g, h]}{\operatorname{tr}^{2} g-4} .
$$

Proof. This follows, up to sign, from the formula in [Jones and Reid 1997] for calculating the distance between the axes of two nonparabolic isometries of $\mathbb{H}^{3}$. Here is an alternative proof (Figure 1):

Conjugate $g$ and $h$ so that in the upper half-space model of $\mathbb{H}^{3}$, axis $g$ has endpoints \pm 1 on the sphere at infinity $S_{\infty}=\mathbb{C} \cup\{\infty\}$ and the common perpendicular to axis $g$ and axis $h g h^{-1}$ is the $z$-axis.

Let the complex length of $g$ be $2 d$. Then

$$
g=\left(\begin{array}{cc}
\cosh d & \sinh d \\
\sinh d & \cosh d
\end{array}\right)
$$

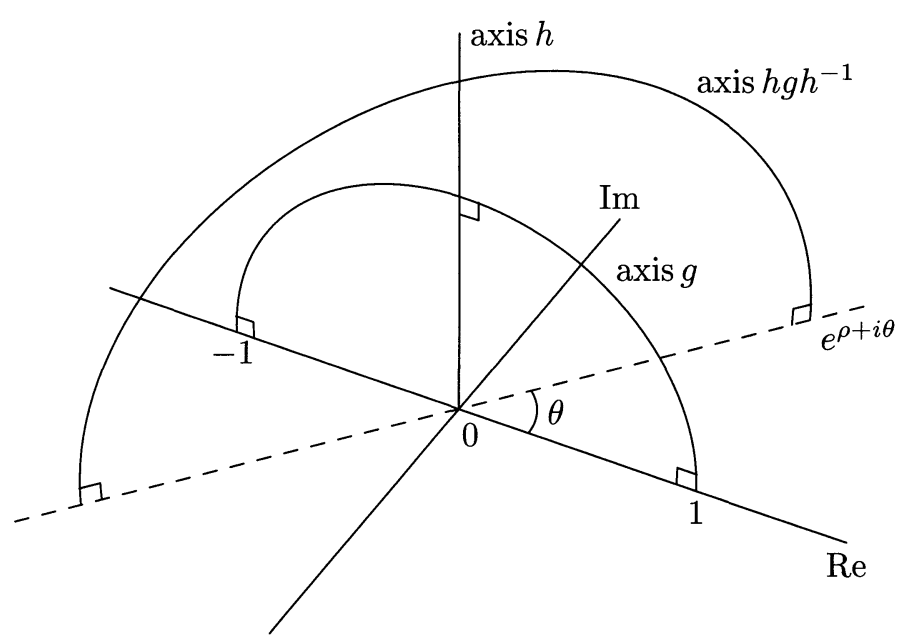

FIGURE 1. The axes of $g$ and $h g h^{-1}$ after suitable conjugation, in the upper half-space model of $\mathbb{H}^{3}$. The axis of the isometry $h$ between them is the $z$-axis. 
The element $h$ corresponds to a composition of two isometries - one with axis the $z$-axis and complex length precisely the complex distance $\rho+i \theta$ between axis $g$ and axis $h g h^{-1}$, and the other with axis the same as axis $g$ and complex length say $2 l$. So we can write

$$
h=\left(\begin{array}{cc}
e^{(\rho+i \theta) / 2} & 0 \\
0 & e^{-(\rho+i \theta) / 2}
\end{array}\right)\left(\begin{array}{cc}
\cosh l & \sinh l \\
\sinh l & \cosh l
\end{array}\right)
$$

and then compute the expression of $[g, h]$ :

$$
\left(\begin{array}{cc}
\cosh ^{2} d-e^{-(\rho+i \theta)} \sinh ^{2} d & \left(1-e^{\rho+i \theta}\right) \cosh d \sinh d \\
\left(1-e^{-(\rho+i \theta)}\right) \cosh d \sinh d & \cosh ^{2} d-e^{\rho+i \theta} \sinh ^{2} d
\end{array}\right) .
$$

This is independent of the second factor of $h$, which commutes with $g$. In particular, $\operatorname{tr} g=2 \cosh d$ and

$$
\begin{aligned}
\operatorname{tr}[g, h] & =2 \cosh ^{2} d-\left(e^{\rho+i \theta}+e^{-(\rho+i \theta)}\right) \sinh ^{2} d \\
& =2 \cosh ^{2} d-2 \cosh (\rho+i \theta) \sinh ^{2} d,
\end{aligned}
$$

so that

$$
\begin{aligned}
& \frac{\operatorname{tr}^{2} g-2 \operatorname{tr}[g, h]}{\operatorname{tr}^{2} g-4} \\
& \quad=\frac{4 \cosh ^{2} d-2\left(2 \cosh ^{2} d-2 \cosh (\rho+i \theta) \sinh ^{2} d\right)}{4 \cosh ^{2} d-4} \\
& \quad=\frac{4 \cosh (\rho+i \theta) \sinh ^{2} d}{4 \sinh ^{2} d}=\cosh (\rho+i \theta) .
\end{aligned}
$$

Lemma 2.2 gives the following nice geometric result.

Lemma 2.3. Let $\gamma$ be a closed geodesic in a hyperbolic three-orbifold $\mathbb{H}^{3} / \Gamma$, corresponding to the axis of $g \in$ $\Gamma \subset \operatorname{PSL}(2, \mathbb{C})$. Then $\gamma$ is nonsimple if and only if there is some $h \in \Gamma$ such that $\operatorname{tr}[g, h]$ lies on the open line segment in the complex plane between 2 and $\operatorname{tr}^{2} g-2$.

Proof. The geodesic $\gamma$ is nonsimple if and only if there is a $h \in \Gamma$ such that in $\mathbb{H}^{3}$, axis $g$ intersects, but is not equal to, its translate $h$ axis $g=\operatorname{axis} h g h^{-1}$. This means that the complex distance $\rho+i \theta$ between these axes has $\rho=0$, or equivalently that $\cosh (\rho+$ $i \theta) \in[-1,1] \subset \mathbb{R}$. The cases $\cosh (\rho+i \theta)= \pm 1$ correspond to axis $h g h^{-1}=$ axis $g$ so are ignored. So, by Lemma $2.2, \gamma$ is nonsimple if and only if

$$
t:=\frac{\operatorname{tr}^{2} g-2 \operatorname{tr}[g, h]}{t r^{2} g-4} \in(-1,1),
$$

or, after rearranging, if and only if

$\operatorname{tr}[g, h]=\frac{1}{2}\left(\operatorname{tr}^{2} g-t\left(\operatorname{tr}^{2} g-4\right)\right), \quad$ with $-1<t<1$.
The right-hand side defines the open straight line segment in the complex plane between 2 and $\operatorname{tr}^{2} g-$ 2 , giving the result.

Proof of Proposition 2.1. Consider a closed geodesic $\gamma$ in $M=\mathbb{H}^{3} / \Gamma$, the axis of $g \in \Gamma$. By Lemma 2.3, and since all traces of elements of $\Gamma \subset \Gamma_{0}=\operatorname{PSL}\left(2, \mathcal{O}_{d}\right)$ lie in this ring of integers $\mathcal{O}_{d}$ in $\mathbb{Q}(\sqrt{-d}), \gamma$ is nonsimple if and only if there is some $h \in \Gamma$ such that $\operatorname{tr}[g, h]$ lies some rational fraction of the distance along the open line segment between 2 and $\operatorname{tr}^{2} g-2$ in the complex plane. Equivalently, by a translation, $\operatorname{tr}[g, h]-2 \in \mathcal{O}_{d}$ must occur some rational fraction of the distance along the open line segment between 0 and $\operatorname{tr}^{2} g-4$, say

$$
\operatorname{tr}[g, h]-2=\frac{m}{n}\left(\operatorname{tr}^{2} g-4\right)
$$

for some $m, n$ integers such that $n>1$ and $0<m<$ $n$. In particular, if $\gamma$ is nonsimple, some integer $n>1$ divides $\operatorname{tr}^{2} g-4$. Since $\operatorname{tr} g \in \mathcal{O}_{d}$, it can be written in the form $\operatorname{tr} g=a+b \omega$ for some $a, b \in \mathbb{Z}$ (where $\omega$ is defined in the beginning of this section), so that

$$
\operatorname{tr}^{2} g-4=a^{2}+2 a b \omega+b^{2} \omega^{2}-4 .
$$

We consider the two possible cases for $\omega$ separately.

Case $1 . \quad d \equiv-1 \bmod 4$, so $\omega=\frac{1}{2}(1+\sqrt{-d})$. Let $d=-1+4 l, l \in \mathbb{N}$. Then $\omega^{2}=\omega-l$, and $(2-1)$ becomes

$$
\begin{aligned}
\operatorname{tr}^{2} g-4 & =a^{2}+2 a b \omega+b^{2}(\omega-l)-4 \\
& =\left(a^{2}-l b^{2}-4\right)+\left(2 a b+b^{2}\right) \omega .
\end{aligned}
$$

So, if $\gamma$ is nonsimple there is some integer $n>1$ such that $n$ divides $\left(a^{2}-l b^{2}-4\right)+\left(2 a b+b^{2}\right) \omega$, and thus $n$ divides $\operatorname{gcd}\left(a^{2}-l b^{2}-4,2 a b+b^{2}\right)$. Suppose $a=0$ and $b$ is odd. Then

$$
\operatorname{gcd}\left(a^{2}-l b^{2}-4,2 a b+b^{2}\right)=\operatorname{gcd}\left(-l b^{2}-4, b^{2}\right),
$$

where $b^{2}$ is odd. Since $2 \nmid b^{2}$, and since any other prime divisor of $b^{2}$ divides $l b^{2}$ also but not 4 , we have $\operatorname{gcd}\left(-l b^{2}-4, b^{2}\right)=1$. So $\gamma$ is simple for this $a$ and $b$.

Case 2 . $d \equiv 1$ or $2 \bmod 4$, so $\omega=\sqrt{-d}$. Here $\omega^{2}=$ $-d$, so that $(2-1)$ becomes

$$
\begin{aligned}
\operatorname{tr}^{2} g-4 & =a^{2}+2 a b \omega+b^{2}(-d)-4 \\
& =\left(a^{2}-d b^{2}-4\right)+(2 a b) \omega .
\end{aligned}
$$


So, if $\gamma$ is nonsimple there is some integer $n>1 \mathrm{such}$ that $n$ divides $\left(a^{2}-d b^{2}-4\right)+(2 a b) \omega$, which means $n$ divides $\operatorname{gcd}\left(a^{2}-d b^{2}-4,2 a b\right)$. Take $a=1$ and $b \equiv 2$ or $4 \bmod 6$. Then $\operatorname{gcd}\left(a^{2}-d b^{2}-4,2 a b\right)=$ $\operatorname{gcd}\left(-d b^{2}-3,2 b\right)$ where $b^{2}$ is even and $3 \nmid b$. Since $2 \nmid$ $\left(-d b^{2}-3\right)$ and $3 \nmid 2 b$, and moreover any larger prime divisor of $2 b$ also divides $d b^{2}$ but not 3 , we have $\operatorname{gcd}\left(-d b^{2}-3,2 b\right)=1$. So again, for this $a$ and $b, \gamma$ is simple.

In either case, therefore, we can find infinitely many elements of $\Gamma_{d}$ whose corresponding geodesics in $M_{d}=\mathbb{H}^{3} / \Gamma_{d}$ are simple - for example, elements of the form

$$
g_{j}=\left(\begin{array}{rr}
0 & 1 \\
-1 & c_{j}
\end{array}\right) \quad \text { for } j \in \mathbb{Z},
$$

where

$$
c_{j}= \begin{cases}(2 j+1) \omega & \text { in case } 1 \\ 1+(6 j+2) \omega & \text { in case } 2 .\end{cases}
$$

However, we would like infinitely many distinct simple geodesics to occur in this way. But elements $g_{j_{1}}$ and $g_{j_{2}}$ give the same primitive geodesic precisely when $g_{j_{1}}=g^{m}$ and $g_{j_{2}}=h g^{n} h^{-1}$ for some $g, h \in \Gamma_{d}$ and $m, n \in \mathbb{Z} \backslash\{0\}$. The traces $\operatorname{tr} g_{j_{1}}$ and $\operatorname{tr} g_{j_{2}}$ then arise as traces of powers of a common element $g \in \Gamma_{d}$. So it suffices to show that the infinite set of traces $\left\{\operatorname{tr} g_{j}\right\}_{j \in \mathbb{Z}}$ for these simple geodesics do not all occur as traces of powers of a finite set of elements of $\Gamma_{d}$.

For a fixed element $g$, with eigenvalues $\lambda^{ \pm 1}$ where $|\lambda|>1$, we have $\left|\operatorname{tr} g^{N}\right|=\left|\lambda^{N}+\lambda^{-N}\right| \approx|\lambda|^{N}$ for $N$ large. So the series $\sum_{n} 1 /\left|\operatorname{tr} g^{n}\right|$ is approximately a geometric series with ratio $1 /|\lambda|<1$, and thus converges. Hence for any set of traces $\left\{\operatorname{tr}_{i}\right\}_{i \in I}$ expressible as traces of powers of a finite set of elements, the $\operatorname{sum} \sum_{i \in I} 1 /\left|\operatorname{tr}_{i}\right|$ is finite. In our case, however, since the traces $\left\{\operatorname{tr} g_{j}\right\}_{j \in \mathbb{Z}}=\left\{c_{j}\right\}_{j \in \mathbb{Z}}$ form an arithmetic progression as at $(2-2)$, the series $\sum_{j \in \mathbb{Z}} 1 /\left|\operatorname{tr} g_{j}\right|$ diverges.

So there are indeed infinitely many distinct simple closed geodesics in $M_{d}=\mathbb{H}^{3} / \Gamma_{d}$. For $\Gamma$ a finite index subgroup of $\Gamma_{d}, M=\mathbb{H}^{3} / \Gamma$ is a finite-sheeted cover of $M_{d}$, and so these geodesics lift to distinct simple closed geodesics in $M$ also.

It is proved in [Miller $\geq 2001$ ] that every cusped hyperbolic three-manifold contains infinitely many simple closed geodesics. This provides large classes of hyperbolic three-manifolds containing many geodesic knots. We next discuss topological restrictions on their complements.

Define the Fox group of a knot $k$ in a three-manifold $M$ to be

$$
G=\operatorname{ker}\left(i_{*}: \pi_{1}(M-k) \rightarrow \pi_{1}(M)\right),
$$

where $i_{*}$ is induced by the inclusion map $i: M-k \rightarrow$ $M$. This is a fundamental invariant of the knot.

Theorem 2.4 [Sakai 1991]. The Fox group of a simple closed geodesic in an orientable hyperbolic threemanifold is a free group.

While Sakai sates this and subsequent results in his paper with the added supposition that the manifold be closed, his proofs hold also in the noncompact case, so we present them here in this more general form.

Theorem 2.5 [Sakai 1991]. Suppose that $M$ is an orientable simple three-manifold whose universal cover is $\mathbb{R}^{3}$. Let $\alpha$ be a noncontractible knot in $M$ such that

(1) $\alpha$ is primitive (that is, if $\alpha$ is freely homotopic to $\beta^{p}$ for some loop $\beta$ and an integer $p$, then $p=$ $\pm 1)$, and

(2) $G=\operatorname{ker}\left(i_{*}: \pi_{1}(M-\alpha) \rightarrow \pi_{1}(M)\right)$ is a free group.

Then $\alpha$ is a simple knot in $M$.

Recall that a knot is simple if its complement contains no essential tori.

Theorems 2.4 and 2.5, together with Thurston's Uniformization Theorem, then yield:

Theorem 2.6 [Sakai 1991]. A geodesic knot in an orientable hyperbolic three-manifold is a hyperbolic knot.

This is extended to simple geodesic links in [Kojima 1988].

We will refer to the conditions of having free Fox group and being hyperbolic as Sakai's conditions on a geodesic knot in a hyperbolic three-manifold.

The final topological result we quote is a proposition of J. Dubois, which incorporates Theorem 2.4. Again, the restriction to the case of closed manifolds is unnecessary, so we do not impose it here. 
Proposition 2.7 [Dubois 1998]. The following knots in three-manifolds all have free Fox group:

(i) simple closed geodesics in hyperbolic manifolds,

(ii) fibres of Seifert manifolds,

(iii) a knot transverse to each fibre of a manifold fibred over the circle,

(iv) a simple closed curve in an incompressible surface of a Haken manifold,

(v) a knot isotopic via an embedded annulus to a simple closed curve in the boundary of an irreducible compact manifold,

(vi) a simple closed curve contained in the set of double points of a surface which is orientable, immersed, $\pi_{1}$-injective and has the "1-line and 3 planes intersection property" considered by Hass and Scott.

So, any knots of type (iii), (iv), (v) or (vi) in an orientable hyperbolic three-manifold satisfy the conclusion of Sakai's Theorem 2.4. However, in case $(\mathrm{v})$, if the manifold has torus boundary, the complement of the knot contains an essential torus, and therefore is not hyperbolic. So the three knot classes (iii), (iv) and (vi) are candidates for providing sufficient topological conditions on a hyperbolic knot for it to be isotopic to a geodesic. Later, in Section 4, we study further the case (iii) of knots transverse to each fibre of a manifold fibred over the circle.

\section{Computer Programs Used}

Jeff Weeks' program SnapPea [1993] and the exact version Snap by Oliver Goodman [Coulson et al. 2000; Goodman et al. 1998] will be used widely henceforth. These programs compute hyperbolic structures following drilling on three-manifolds, and can provide a variety of associated topological, geometric and arithmetic invariants. Most useful for our purposes will be hyperbolic volume and core geodesic length (following Dehn filling). SnapPea can also determine whether two given manifolds are isometric via its 'isometry checker'. Snap can list all closed geodesics up to a given length in a manifold by their free homotopy class, and then determine to which of these classes any given word belongs. Goodman's related program Tube [Goodman et al. 1998] allows us to view these geodesics in a Dirichlet domain for the manifold using Geomview [Phillips et al. 1993]. It also provides the tube radius of each geodesic (the radius of the largest embedded tubular neighbourhood of the geodesic), and can drill any such simple geodesic from the manifold, using a technique developed in [Dowty 2000].

\section{GEODESICS IN THE FIGURE-EIGHT KNOT COMPLEMENT}

The rest of this paper is devoted to the case of the figure-eight knot complement. In order to study topological properties of its geodesics, we first develop methods of locating them up to isotopy. Then, using the software just discussed, we can drill them and get topological information about them.

\section{A Useful Presentation of the Fundamental Group}

Thurston [1997, pp. 39-42, 128-129] demonstrates how to obtain an ideal triangulation of the figureeight knot complement, starting by picturing the manifold 'in $S^{3}$, as the complement of the projection of the figure-eight knot $k$ shown in Figure 2,
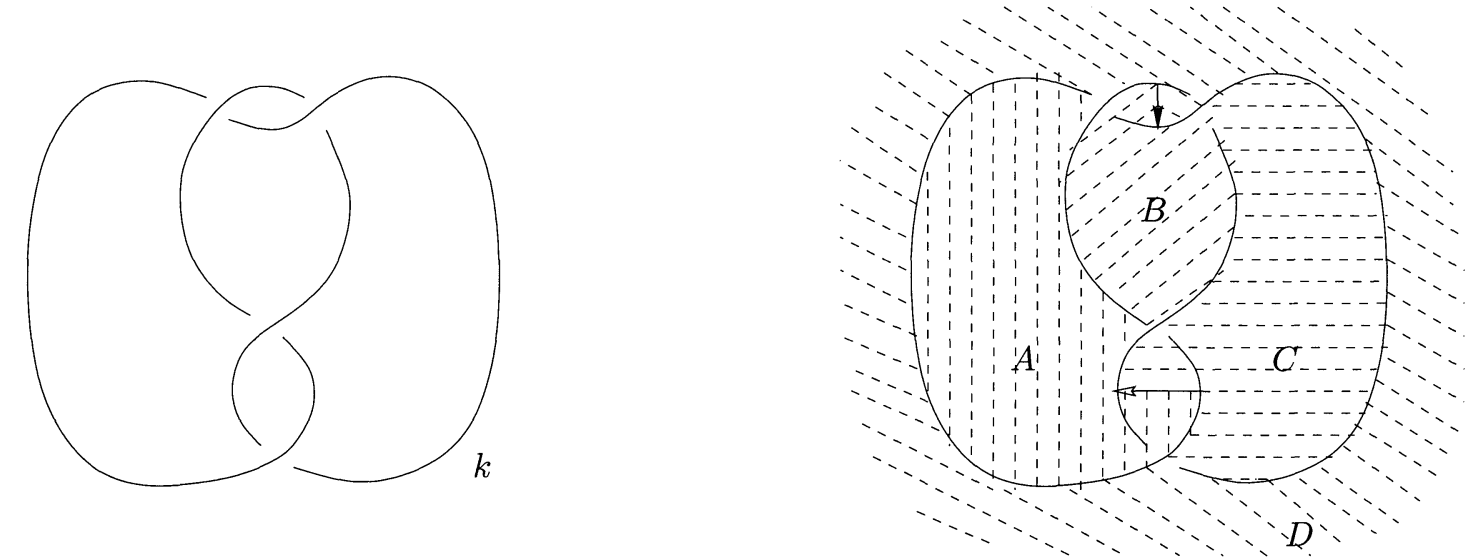

FIGURE 2. Spanning the figure-eight knot by a 2-complex. 
left. To this projection of $k$, we firstly introduce two additional edges (shown as arrows) and then span by a 2-complex with four faces $A, B, C, D$, as in Figure 2, right. The 2-complex is the boundary of two balls in $S^{3}$, which become the two ideal tetrahedra of the triangulation, subject to the identifications induced by the common boundary sphere, as pictured in Figure 3.

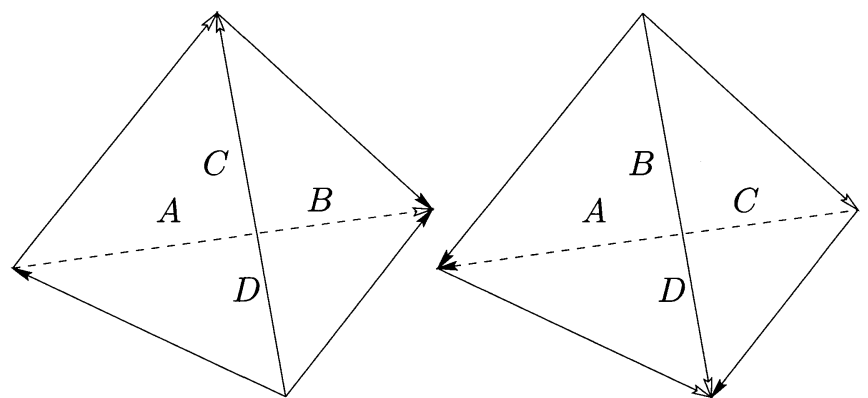

FIGURE 3. The tetrahedra on each side of the 2-complex that defines the triangulation. Letters are centered on the faces they label, and faces with the same letter are to be identified in such a way that arrows match.

The generators SnapPea obtains for the fundamental group correspond to loops dual to this triangulation, and we can trace these back to loops in the $S^{3}$ view of the manifold, as in Figure 4.

This gives a direct way of converting between a simple closed curve drawn in this $S^{3}$ view of the knot complement, and its homotopy class represented as a word in the generators $a, b, c$ of the fundamental group.

A presentation for the fundamental group with these generators is

$$
\pi_{1}=\left\langle a, b, c \mid c a^{-1} b c^{-1} a=1, a b^{-1} c^{-1} b=1\right\rangle .
$$

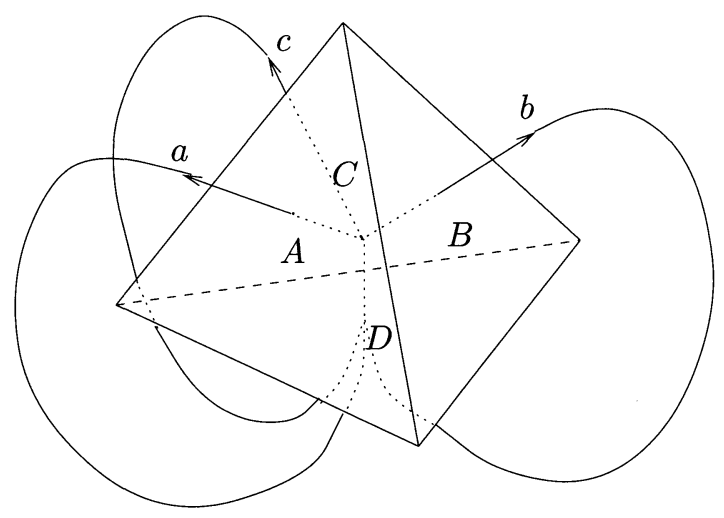

\section{Looking at Geodesics in $\mathrm{S}^{3}-\mathrm{k}$}

Table 1 lists all geodesics up to length 2.9 in the complement of the figure-eight knot $k$, as obtained by Snap. By the preceding discussion we can draw closed curves which are freely homotopic, but not necessarily isotopic, to these geodesics. We would like to know when we have found the true geodesic, and there are various ways of doing so.

So, suppose we have drawn a curve $c$ freely homotopic to geodesic $\gamma$ in $S^{3}-k$.

Method 1. Use SnapPea to drill $c$ from $S^{3}-k$. Perform $(1,0)$-Dehn filling on this $c$ cusp to recover the figure-eight knot complement. If there are no negatively oriented ideal tetrahedra in SnapPea's resulting triangulation, the core of this filling, $c$, was geodesic [Thurston 1979, Chapter 4].

While this method is straight-forward, its disadvantage is that the converse to the final statement does not hold - there is no guarantee that a true geodesic will yield only positively oriented ideal tetrahedra and thus be found. In practice, this method is useful in only a small number of cases.

Method 2. Use Tube to drill $\gamma$ from $S^{3}-k$, and SnapPea as before to drill $c$ from $S^{3}-k$. If SnapPea's isometry checker finds that the resulting manifolds match by an isometry taking meridian loops to meridian loops, then the links $k \cup \gamma$ and $k \cup c$ are isotopic, and hence $c$ isotopic to the geodesic $\gamma$ in $S^{3}-k$.

For short geodesics, where the correct isotopy class should be found in few attempts, this method can

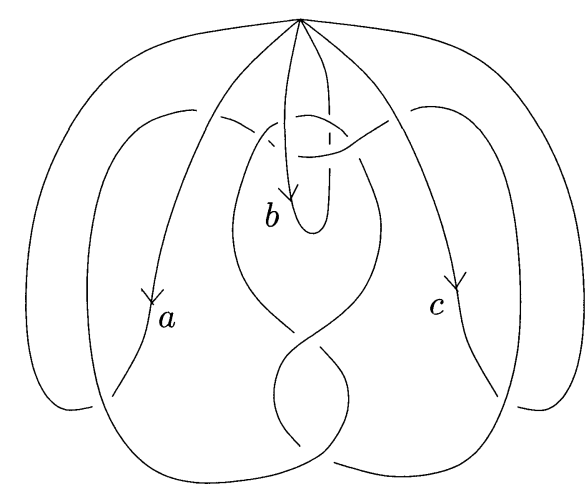

FIGURE 4. Generators of $\pi_{1}\left(S^{3}-k\right)$, as loops dual to the triangulation (left), and in the $S^{3}$ view (right). 


\begin{tabular}{|c|c|c|c|}
\hline \# & complex length & tube rad. & class \\
\hline 0 & $1.087070-1.722768 i$ & 0.426680 & $B$ \\
\hline 1 & $1.087070+1.722768 i$ & 0.426680 & $A c$ \\
\hline 2 & $1.662886-2.392124 i$ & 0.274653 & $A B$ \\
\hline 3 & $1.662886+2.392124 i$ & 0.274653 & $A^{2} c$ \\
\hline 4 & $1.662886-2.392124 i$ & 0.274653 & $B^{2} c$ \\
\hline 5 & $1.662886+2.392124 i$ & 0.274653 & $\mathrm{CaC}$ \\
\hline 6 & $1.725109-0.921839 i$ & 0.211824 & $C A$ \\
\hline 7 & $1.725109+0.921839 i$ & 0.211824 & $A C b$ \\
\hline 8 & $2.174140-2.837648 i$ & 0.187120 & $A^{2} B$ \\
\hline 9 & $2.174140+2.837648 i$ & 0.187120 & $A^{3} c$ \\
\hline 10 & $2.174140-2.837648 i$ & 0.187120 & $B^{2} a c$ \\
\hline 11 & $2.174140+2.837648 i$ & 0.187120 & $\mathrm{CaC} C^{2}$ \\
\hline 12 & $2.416113-1.208686 i$ & 0.111840 & $C A^{2}$ \\
\hline 13 & $2.416113-1.208686 i$ & 0.111840 & $B a c^{2}$ \\
\hline 14 & $2.416113+1.208686 i$ & 0.111840 & $B c^{3}$ \\
\hline 15 & $2.416113+1.208686 i$ & 0.111840 & $A C b C b$ \\
\hline 16 & $2.633916+0.000000 i$ & 0.271768 & $B c a c$ \\
\hline 17 & $2.633916+3.141593 i$ & 0.000000 & $A B^{2} c$ \\
\hline 18 & $2.633916+3.141593 i$ & 0.127639 & $C A^{2} B$ \\
\hline 19 & $2.633916+3.141593 i$ & 0.000000 & $C a^{2} C$ \\
\hline 20 & $2.633916+3.141593 i$ & 0.127639 & $A^{3} c A$ \\
\hline 21 & $2.633916+3.141593 i$ & 0.127639 & $B^{2} a c^{2}$ \\
\hline 22 & $2.633916+3.141593 i$ & 0.127639 & $B c^{3} A c$ \\
\hline 23 & $2.839470+2.192690 i$ & 0.022793 & $A B^{2}$ \\
\hline 24 & $2.839470-2.192690 i$ & 0.179924 & $A C B C$ \\
\hline 25 & $2.839470+2.192690 i$ & 0.179924 & $B A c^{2}$ \\
\hline 26 & $2.839470+2.192690 i$ & 0.022793 & $B^{3} c$ \\
\hline 27 & $2.839470+2.192690 i$ & 0.179924 & $B a^{2} C$ \\
\hline 28 & $2.839470-2.192690 i$ & 0.022793 & $A^{2} c A c$ \\
\hline 29 & $2.839470-2.192690 i$ & 0.179924 & $A B^{2} c^{2}$ \\
\hline 30 & $2.839470-2.192690 i$ & 0.022793 & $A c^{2} A c$ \\
\hline
\end{tabular}

TABLE 1. Geodesics in the figure-eight knot complement. Shown are the number assigned to the geodesic, the complex length, the tube radius and the free homotopy class (where we use $A, B, C$ for $a^{-1}$, $b^{-1}, c^{-1}$.)

be quite efficient. For long geodesics, however, it is not practical.

Method 3. Use Tube to view the geodesic $\gamma$ in a Dirichlet domain for $S^{3}-k$ via Geomview [Phillips et al. 1993], then convert back to the $S^{3}$ picture.

This method eliminates guesswork and also handles all closed geodesics, including those with selfintersection, but becomes very difficult to implement for long $\gamma$.

Using the methods above we can therefore determine pictures of the geodesics in Table 1 up to isotopy. By making use of the symmetry group of the manifold, we can reduce the work needed, since isometries map geodesics to geodesics. Sets of geodesics in Table 1 with the same length and tube radius are related by isometries from this symmetry group, the dihedral group

$$
D_{4}=\left\langle x, y \mid x^{2}=1, y^{4}=1, x^{-1} y x=y^{-1}\right\rangle .
$$

The action of this group can be seen from the more symmetric picture of the figure-eight knot $k$ shown in Figure 5.

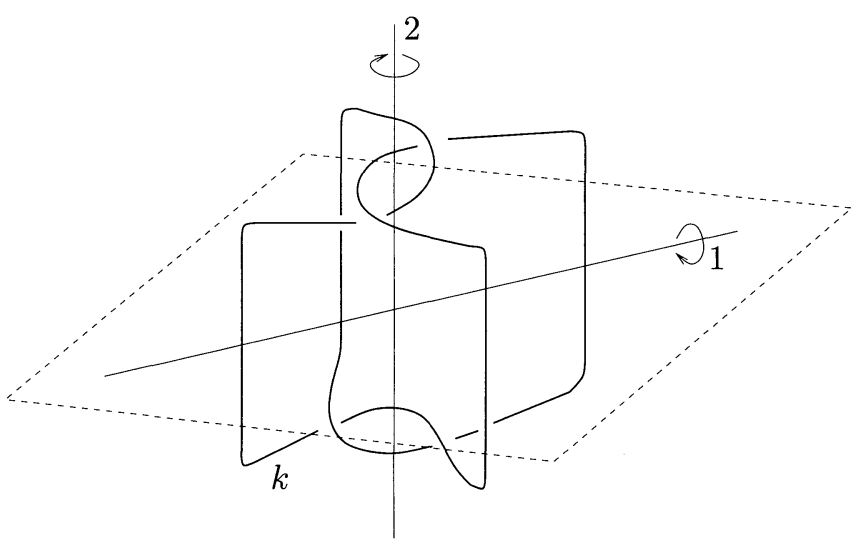

FIGURE 5. Action of the symmetry group on $S^{3}-k$. Here $x$ is rotation by $\pi$ about axis 1 followed by inversion in the box-like region $k$ encloses, and $y$ is rotation by $\pi / 2$ about axis 2 followed by reflection across the horizontal plane.

Table 2 on the next page lists all geodesics in this manifold with real length up to 3.65 by their symmetry orbits, as determined by Tube. It also shows their tube radii, and if simple, their complement volume and knot type in $S^{3}$. Note that even geodesics as low as number 23 are nontrivially knotted. Figure 6 shows examples, up to isotopy, of geodesics of each of the three nontrivial knot types occuring in Table 2. The two geodesics here of knot types $6_{1}$ and $9_{42}$ are in fact part of an infinite family containing geodesics representing infinitely many different knot types in $S^{3}$ [Miller $\geq 2001$.

Using this table we can choose to find just one geodesic from each symmetry orbit via the methods above, and use the action of the symmetry group to determine the others. Thus Figure 7 shows one geodesic per symmetry orbit, pictured up to isotopy, for all geodesics listed in Table 1. These form a basis for our observations about geodesics amongst homotopic closed curves. 


\begin{tabular}{|c|cccc|cccc|}
\hline complex length & tube rad. & geod. \#s & volume & kn. & tube rad. & geod. \#s & volume & kn. \\
\hline $1.087070+1.722768 i$ & 0.426680 & 0,1 & 3.663862 & 0 & & & & \\
$1.662886+2.392124 i$ & 0.274653 & $2,4,5,3$ & 4.415332 & 0 & & & & \\
$1.725109+0.921839 i$ & 0.211824 & 6,7 & 5.333490 & 0 & & & & \\
$2.174140+2.837648 i$ & 0.187120 & $8,10,11,9$ & 5.137941 & 0 & & & & \\
$2.416113+1.208686 i$ & 0.111840 & $12,13,14,15$ & 6.290303 & 0 & & & & \\
$2.633916+0.000000 i$ & 0.271768 & 16 & 8.119533 & 0 & & & & \\
$2.633916+3.141593 i$ & 0.000000 & 17,19 & not simple & 0.127639 & $18,21,22,20$ & 5.916746 & 0 \\
$2.839470+2.192690 i$ & 0.022793 & $23,26,30,28$ & 5.729381 & $3_{1}$ & 0.179924 & $24,29,25,27$ & 8.085587 & 3 \\
$2.921563+1.381744 i$ & 0.071578 & $32,34,35,36$ & 6.770817 & 0 & 0.271246 & 31,33 & 8.706195 & $3_{1}$ \\
$3.040161+2.932500 i$ & 0.088294 & $40,43,44,41$ & 6.551743 & 0 & 0.181292 & $37,42,39,38$ & 7.694923 & $3_{1}$ \\
$3.261210+1.114880 i$ & 0.065182 & $45,46,47,48$ & 9.053177 & $6_{1}$ & & & & \\
$3.275339+0.715139 i$ & 0.132063 & $51,56,53,55$ & 9.250534 & $6_{1}$ & 0.142405 & $49,50,54,52$ & 9.276865 & $3_{1}$ \\
$3.325772+1.498938 i$ & 0.018074 & $64,65,70,69$ & 8.950382 & $9_{42}$ & 0.049991 & $61,66,67,68$ & 7.047485 & 0 \\
& 0.105419 & 57,63 & 8.519184 & 0 & 0.215639 & $58,62,59,60$ & 9.421637 & $3_{1}$ \\
$3.369922+0.334206 i$ & 0.060708 & $75,77,78,76$ & 9.569817 & $6_{1}$ & 0.117776 & $71,72,73,74$ & 9.340971 & 0 \\
$3.395883+2.785465 i$ & 0.062829 & $87,88,89,90$ & 6.930273 & 0 & 0.105235 & $80,83,81,82$ & 11.356526 & 0 \\
& 0.211499 & $79,86,85,84$ & 7.533918 & $3_{1}$ & & & & \\
$3.450219+1.843678 i$ & 0.035793 & $91,96,93,92$ & 9.401392 & 0 & 0.093730 & $94,95,98,97$ & 8.740307 & $9_{42}$ \\
$3.525494+0.000000 i$ & 0.000000 & 99,101 & not simple & 0.000000 & $100,102,103,104$ & not simple \\
$3.612317+2.140511 i$ & 0.038554 & $109,110,111,112$ & 8.826031 & $9_{42}$ & 0.197080 & $105,108,107,106$ & 9.772337 & $3_{1}$ \\
\hline
\end{tabular}

TABLE 2. Symmetry orbits of geodesics in the figure-eight knot complement (grouped by complex length). For each orbit we give the tube radius, the numbers assigned to the geodesics in the orbit, the hyperbolic volume of the geodesic complement and the knot type.

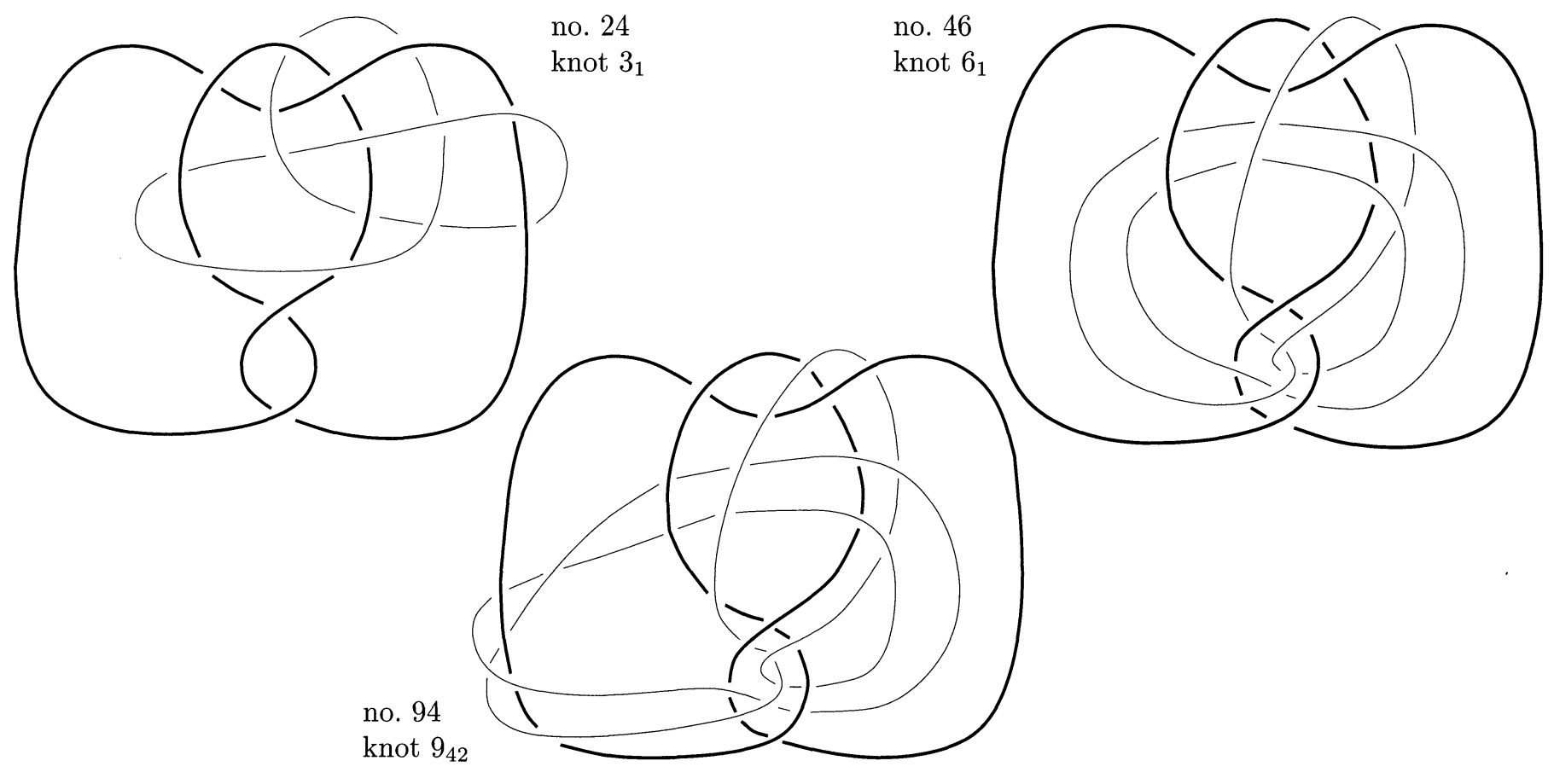

FIGURE 6. Some geodesics in the figure-eight knot complement with nontrivial knot type in $S^{3}$. 


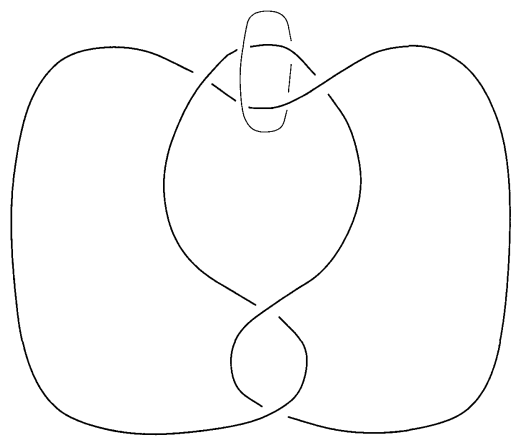

$0 \quad \mathrm{vol}=3.663862$

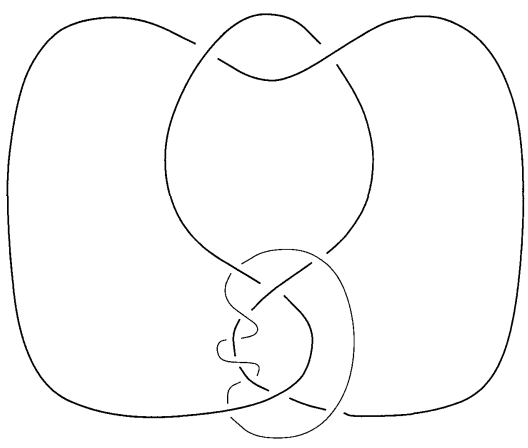

$8 \quad$ vol $=5.137941$

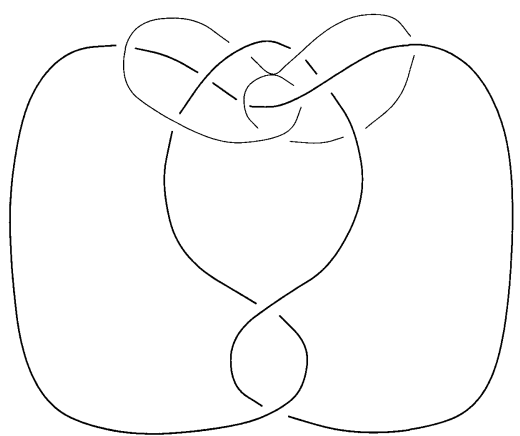

17 nonsimple

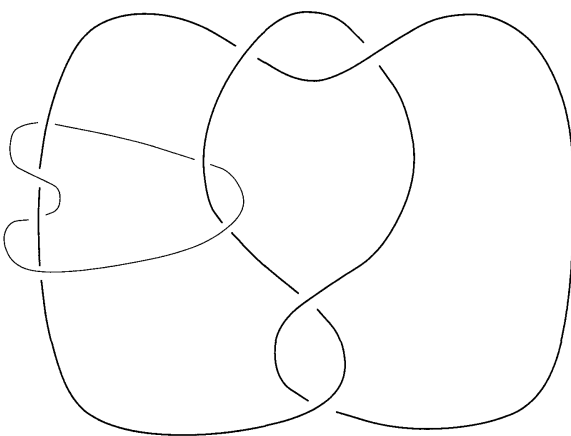

$2 \quad$ vol $=4.415332$

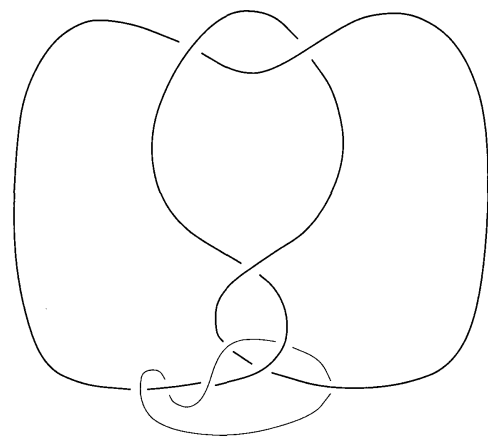

$12 \mathrm{vol}=6.290303$

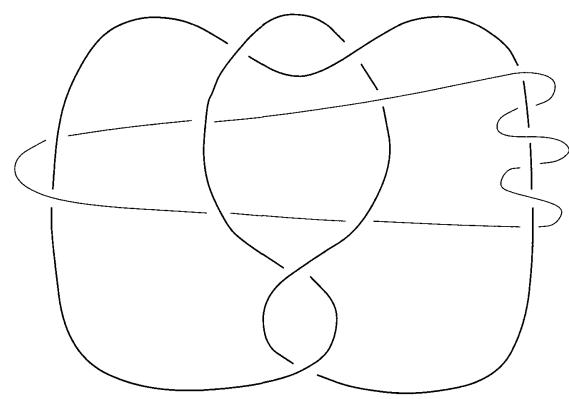

$18 \quad \mathrm{vol}=5.916746$

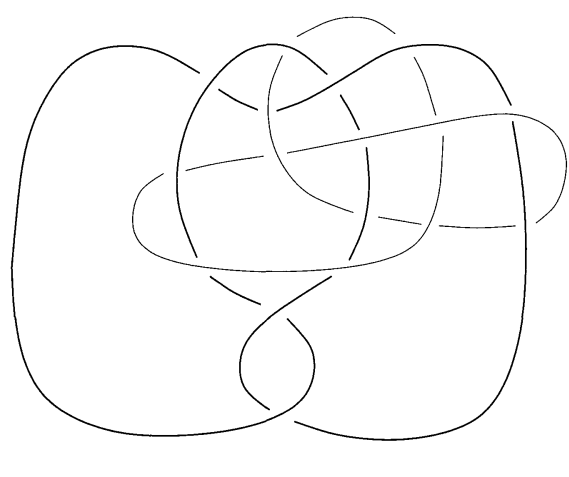

$24 \quad$ vol $=8.085587$

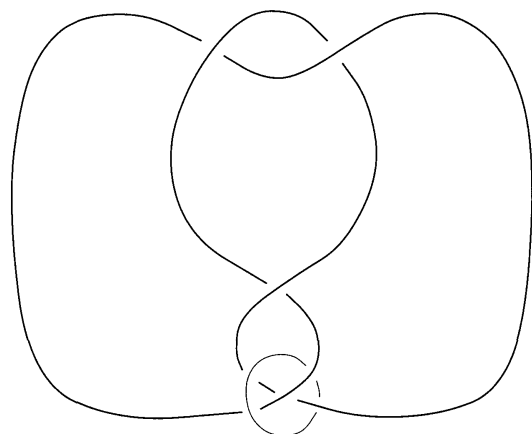

$6 \quad \mathrm{vol}=5.333490$

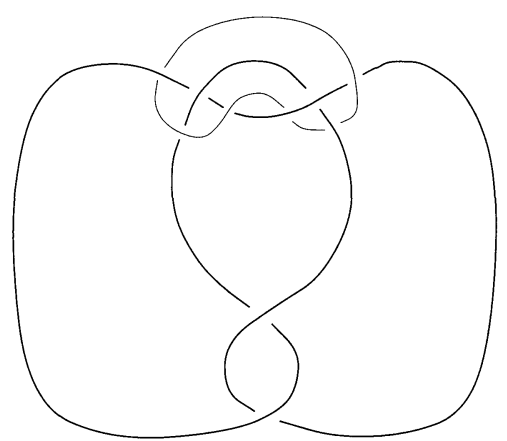

$16 \quad \mathrm{vol}=8.119533$

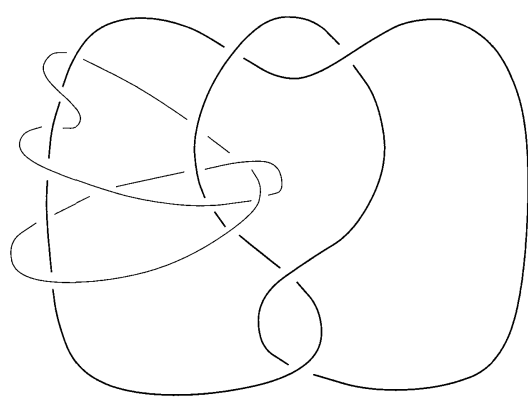

$23 \quad \mathrm{vol}=5.729381$

FIGURE 7. Geodesic symmetry orbit representatives and hyperbolic volume of their complements. 

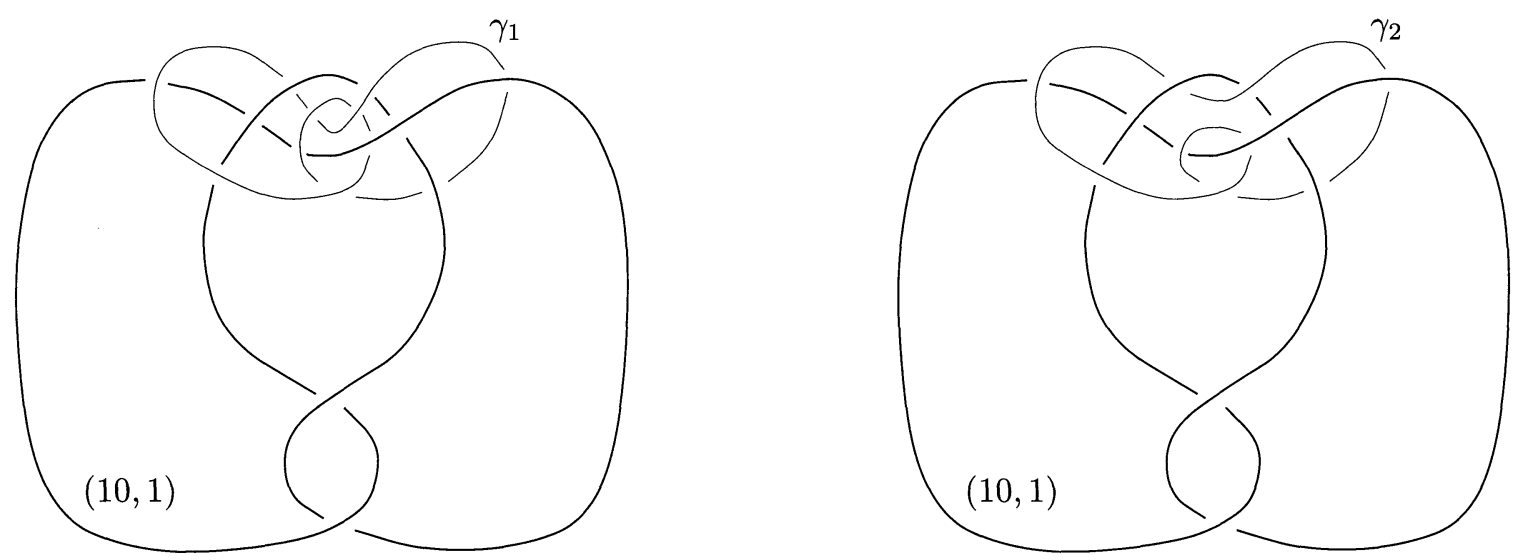

FIGURE 8. Homotopic curves $\gamma_{1}$ and $\gamma_{2}$ in the figure-eight knot complement; $\gamma_{1}$ has lower-volume complement. After performing $(10,1)$ Dehn filling on the figure-eight component, $\gamma_{2}$ becomes the geodesic in the homotopy class, but maintains its higher complement volume.

\section{Findings}

One observation from determining these geodesics and their complements is that the geodesic often appears to have the lowest-volume complement of all curves in its free homotopy class. However, this is not always the case. There exist simple closed geodesics in hyperbolic three-manifolds that do not have the least-volume complement over all curves in their free homotopy class.

Example 3.1. Let $M$ be the figure-eight knot complement and $M(10,1)$ the manifold obtained from it by $(10,1)$ Dehn filling. Then for the homotopic curves $\gamma_{1}$ and $\gamma_{2}$ shown in Figure $8, \gamma_{2}$ is the geodesic but $\operatorname{vol}\left(M(10,1)-\gamma_{1}\right)=6.55462<9.20339=$ $\operatorname{vol}\left(M(10,1)-\gamma_{2}\right)$.

Remark 3.2. Infinite families of such examples can be constructed as follows. Let $M$ be a cusped hyperbolic three-manifold and $\gamma_{0}$ a nonsimple geodesic in $M$ with a single point of self-intersection. Then $\gamma_{0}$ can be perturbed slightly near its self-intersection in two obvious ways, to yield two different knots $\gamma_{1}$ and $\gamma_{2}$ in $M$. The complements of these knots in $M$ will in general have different hyperbolic volumes, say $V_{1}=\operatorname{vol}\left(M-\gamma_{1}\right)<\operatorname{vol}\left(M-\gamma_{2}\right)=V_{2}$. Now, we can change the hyperbolic structure on $M$ slightly by performing high order Dehn fillings $M(p, q)$ on it [Thurston 1979, Chapter 5]. Each resulting hyperbolic manifold $M(p, q)$ will have a geodesic in the homotopy class of $\gamma_{0}$, and generally this geodesic will be isotopic to $\gamma_{1}$ for infinitely many $(p, q)$ and isotopic to $\gamma_{2}$ for infinitely many $(p, q)$. By choosing $(p, q)$ large so that in $M(p, q)$ the geodesic is isotopic to $\gamma_{2}$, we get $\operatorname{vol}\left(M(p, q)-\gamma_{1}\right) \approx V_{1}<V_{2} \approx$ $\operatorname{vol}\left(M(p, q)-\gamma_{2}\right)$, so that the complement of the nongeodesic $\gamma_{1}$ in $M(p, q)$ has lower volume than the complement of the geodesic $\gamma_{2}$.

In the example shown in Figure $8, \gamma_{0}$ in the figureeight knot complement is nonsimple geodesic 17 in Table 1.

So, while this volume-minimising property does not characterise geodesics in general, perhaps it does under certain additional conditions. We consider this further in Section 4.

Another less precise topological observation about the geodesics in Figure 7 is related to the fact that the volume of the complement of a knot in a manifold is a reasonable indication of the knot's complexity. We observe that a geodesic knot appears to always have a very simple embedding, compared to those of other curves in its homotopy class in our $S^{3}$ view of the figure-eight knot complement. It would be interesting to investigate this notion further, and see if there is a rigorous way of expressing it.

\section{CLOSED ORBITS IN SUSPENSION FLOWS}

Recall that a geodesic knot in a hyperbolic threemanifold satisfies Sakai's conditions, of having free Fox group and hyperbolic complement. In this section, we show that these conditions are not sufficient in general to guarantee that a simple closed curve is geodesic. We also investigate whether they could be sufficient for special classes of knots. In particular, we discuss the case of knots arising as closed or- 

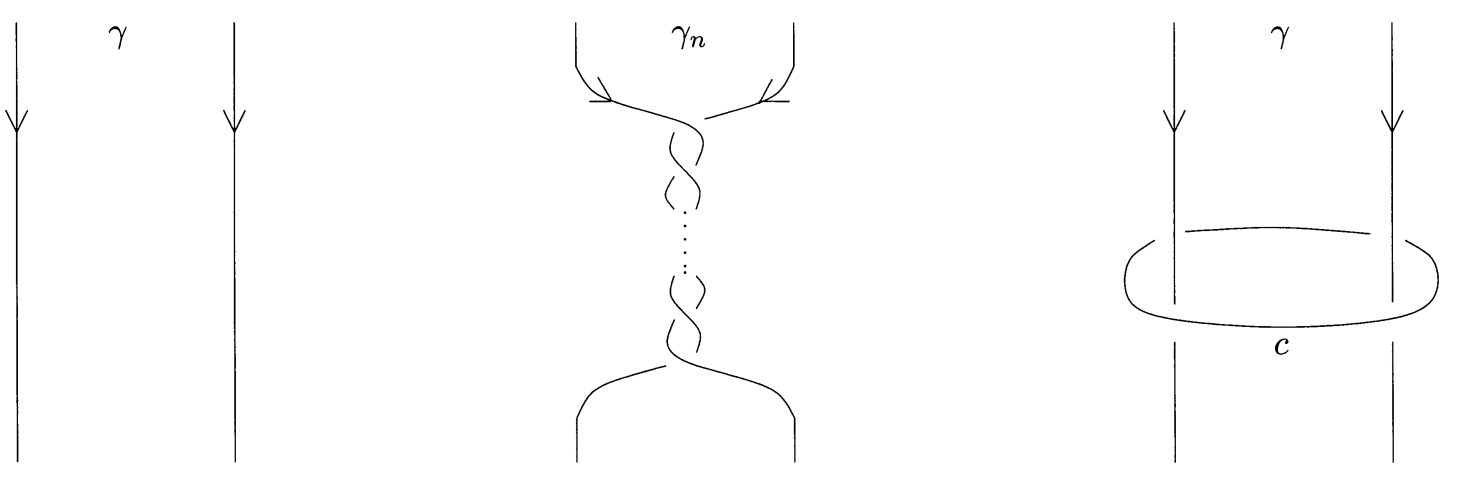

FIGURE 9. The local picture of the geodesic knot $\gamma$ (left) and a homotopic curve $\gamma_{n}$ with $n$ twists (middle), passing transversely through a fibration. The twisting is equivalent to performing $(1, n)$ surgery along curve $c$ (right).

bits in the suspension flow of a manifold fibred over the circle. In the figure-eight knot complement, we study these knots in detail.

\section{Knots Transverse to Fibres}

By Proposition 2.7 we know that a knot transverse to each fibre of a manifold fibred over the circle has free Fox group. Looking at certain families of such knots allows us to prove the following result.

Proposition 4.1. There are infinitely many examples of knots satisfying Sakai's conditions which are not geodesic.

Proof. Consider a hyperbolic knot $\gamma$ which is transverse to each fibre in a hyperbolic three-manifold fibred over the circle and whose period with respect to this fibration is greater than one. Somewhere in the manifold there is then a local picture looking like Figure 9, left, with fibres locally horizontal planes.

We can modify $\gamma$ locally by an integral number $n$ of twists to obtain a family of homotopic but not isotopic curves $\left\{\gamma_{n}\right\}$. By Dubois, each $\gamma_{n}$ still has free Fox group, and moreover, $\gamma_{n}$ will generally be hyperbolic. For, giving $\gamma n$ twists in the direction shown is equivalent to performing $(1, n)$ surgery along a curve $c$ shown in Figure 9, right. Then, using Thurston's hyperbolic Dehn surgery theorem, as long as the result of drilling $c$ is hyperbolic, all manifolds obtained from $(1, n)$ filling on this component for $|n|$ sufficiently large, are also hyperbolic. This gives an infinite family of distinct homotopic curves satisfying Sakai's conditions - only one of which can be geodesic.
For example, the figure-eight knot complement is fibred over the circle with fibre its minimal genus Seifert surface, a punctured torus, as in Figure 10.

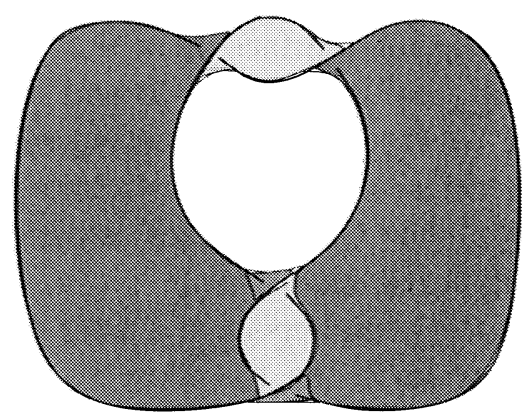

FIGURE 10. A punctured torus fibre in the figure-eight knot complement.

Its geodesic 7 from Snap's list can be drawn to be transverse to each fibre as in the first diagram in Figure 11. Adding a full twist to this curve as in the figure's middle diagram yields a homotopic curve still transverse to each fibre, whose complement can also be obtained from the complement of geodesic 7 by performing $(1,-1)$ surgery along the curve $c$, with hyperbolic complement, shown in Figure 11, right. Since this manifold and all others obtained from $(1, n)$ surgery for low $n$ are hyperbolic, we in fact have that all $(1, n)$ surgeries yield hyperbolic manifolds, giving an infinite family of explicit counterexamples to the converse of Sakai's conditions.

The geodesic in the example above has the special property of being a 'closed orbit' under the 'suspension flow' on the figure-eight knot complement. In the next section we describe how such closed orbits arise in a manifold fibred over the circle, and begin a detailed investigation of them. 

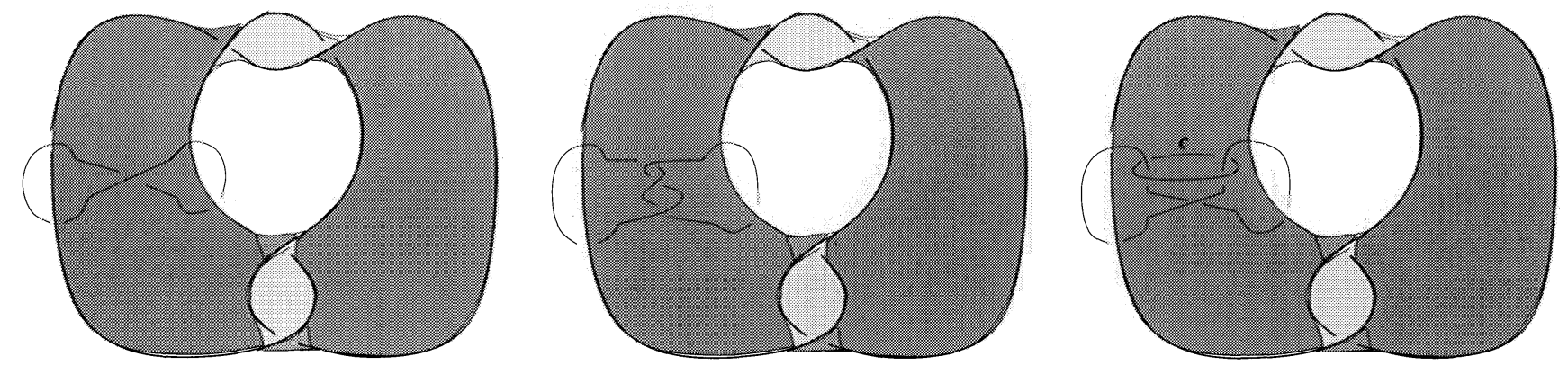

FIGURE 11. A geodesic (left) and a homotopic, nongeodesic, curve (middle) in the figure-eight knot complement, drawn transverse to each fibre of its fibration. The complement of the nongeodesic curve can also be obtained by performing $(1,-1)$ surgery on the curve $c$ shown on the right.

\section{Closed Orbits in the Figure-Eight Knot Complement}

A knot or link $k$ is said to be fibred if its complement $S^{3}-k$ fibres over $S^{1}$, with fibre $F$ orientable and satisfying $\partial F=k$. That amounts to saying that there exists an orientable surface $F=\operatorname{int}(\bar{F})$ for compact $\bar{F}$, with boundary $\partial \bar{F}=k$ and a diffeomorphism $\Phi: F \rightarrow F$ such that $S^{3}-k$ is homeomorphic to the quotient space $F \times I / \Phi$ with identifications $(x, 0)=(\Phi(x), 1)$. The surface $F$ is called the fibre and the map $\Phi$ the monodromy.

In fibring the complement of a knot or link $l$, we induce a flow on it: as the fibre $F$ spins around its boundary $l$ to fill up $S^{3}-l$, each point $x$ in $F$ traces out a path $\{x\} \times I$ in the complement. This particular flow on $S^{3}-l$ considered as the quotient space $F \times I / \Phi$, is called the suspension flow of the monodromy $\Phi$. The knots arising as closed orbits of this flow are of interest to us, as these are transverse to each fibre of the fibration, and hence have free Fox group. They arise from periodic points of $\Phi$, that is, points $x$ in the fibre such that $\Phi^{n}(x)=x$ for some $n$.

Now, the figure-eight knot is fibred with fibre $F$ its minimal genus Seifert surface, a punctured torus, as shown previously in Figure 10. For a detailed description and aid in visualising this fibration, see [Francis 1987]. Viewing this fibre $F$ as the quotient of $\mathbb{R}^{2}$ minus the integer lattice by the action of $\mathbb{Z}^{2}$, the monodromy $\Phi: F \rightarrow F$ is conjugate to the Anosov map induced on this quotient space by the matrix $\left(\begin{array}{ll}2 & 1 \\ 1 & 1\end{array}\right)$ acting on $\mathbb{R}^{2}-\mathbb{Z}^{2}$. So we are interested in the periodic points of such an Anosov matrix $A$ acting on (a subset of) $\mathbb{R}^{2} / \mathbb{Z}^{2}$. Here we can make use of the Lefschetz number $L$ of such a map [Bredon 1993; Brown 1971], and find that the number of fixed points of the linear map $A^{n}$ acting on the torus is
$\left|L\left(A^{n}\right)\right|=\left|2-\operatorname{tr}\left(A^{n}\right)\right|$. For the monodromy of the figure-eight fibration, a simple induction argument shows that

$$
\left(\begin{array}{ll}
2 & 1 \\
1 & 1
\end{array}\right)^{n}=\left(\begin{array}{ll}
F_{2 n+1} & F_{2 n} \\
F_{2 n} & F_{2 n-1}
\end{array}\right),
$$

where $F_{i}$ is the $i$-th Fibonacci number (with $F_{1}=$ $F_{2}=1$ ), so that it is easy to obtain the data given in Table 3.

\begin{tabular}{|rrrr|}
\hline$n=1$ & 1 & 1 & 1 \\
2 & 5 & 4 & 2 \\
3 & 16 & 15 & 5 \\
4 & 45 & 40 & 10 \\
5 & 121 & 120 & 24 \\
6 & 320 & 300 & 50 \\
7 & 841 & 840 & 120 \\
8 & 2205 & 2160 & 270 \\
\hline
\end{tabular}

TABLE 3. For each $n$, the number of points with period dividing $n$ (second column), the number of points with period exactly $n$ (third), and the number of closed orbits with period exactly $n$ (last column).

The single point of period 1 in Table 3 is the point $(0,0)$, corresponding to the puncture in the punctured torus fibre. Thus the number of points with period dividing $n$ as listed in the table is 1 greater than the number we are interested in.

Now, in addition to these closed orbits having free Fox group, we note that:

Proposition 4.2. A closed orbit $c$ in the suspension flow of a hyperbolic three-manifold $M$ fibred over the circle is a hyperbolic knot.

Proof. Let $c$ have period $N$ with respect to the fibration $F \times I / \Phi$ of $M$. Then by drilling $c$ from $M$ we are just removing $N$ points from each fibre $F$ in 
the fibration. Since the pseudo-Anosov monodromy $\Phi$ simply permutes the $N$ points of intersection of $c$ with $F \times\{0\}=F \times\{1\}$, it makes sense to consider the quotient space $F^{\prime} \times I / \Phi$ where $F^{\prime}$ is an $N$-times punctured $F$ and $\Phi$ has been restricted accordingly. Since $\Phi$ remains pseudo-Anosov when restricted to $F^{\prime}$, we then have by Thurston [1986] (see also [Morgan 1984; Otal 1996]), that the manifold $M-c \approx F^{\prime} \times I / \Phi$ is hyperbolic.

Thus these closed orbits in a manifold fibred over $S^{1}$ satisfy Sakai's two conditions for geodesic knots. In an attempt to determine whether they are all geodesic, we now look at ways of describing and analysing them in the case of the figure-eight knot complement.

\section{Templates}

An efficient method of studying closed orbits in flows on three-manifolds is via templates. A template is a compact branched two-manifold with boundary which carries a semiflow (irreversible flow), and which is composed locally of two types of charts joining and splitting — as shown in Figure 12.
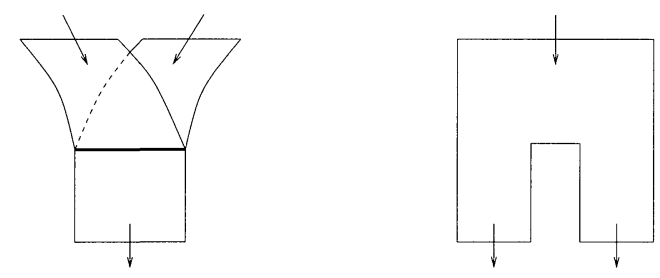

FIGURE 12. A joining chart (left) and a splitting chart (right) for a template.

Our interest in template theory is motivated by the following theorem.

The Template Theorem [Birman and Williams 1983]. Given a flow on a three-manifold $M$ having a hyperbolic chain-recurrent set, there is a template $T \subset M$ such that, with perhaps one or two specified exceptions, the closed orbits under the flow on $M$ are in one-one correspondence with the closed orbits under the semiflow on T. Further, on any finite collection of closed orbits, this correspondence can be taken to be via ambient isotopy.

In particular this holds for the suspension flow of a pseudo-Anosov diffeomorphism of a surface. For the case of this flow on the figure-eight knot complement, we have the template shown in Figure 13,

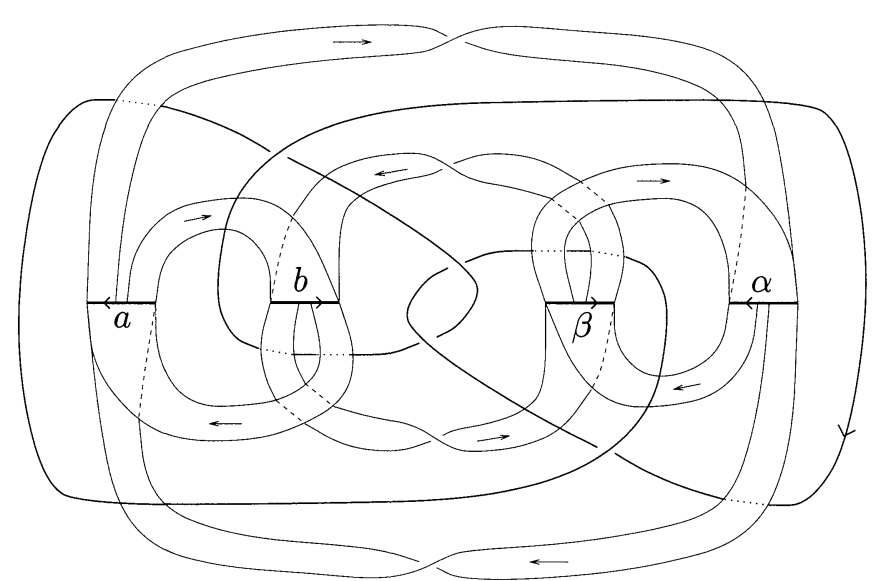

FIGURE 13. A template for the flow on the complement of the figure-eight knot $k$, with branch lines labelled for a symbolic dynamics description.

which was constructed via branched coverings in [Birman and Williams 1983].

Orbits lying on a template can be conveniently described via symbolic dynamics. Here, we first assign symbols $\alpha, \beta, a, b$ to the four branch lines as shown. The template can then be divided into eight 'strips', corresponding to the eight different paths possible between two different branch lines - namely $\alpha \beta, \alpha a$, $\beta \alpha, \beta b, a b, a \alpha, b a$ and $b \beta$. An infinite word $u=$ $u_{0} u_{1} u_{2} \ldots$ in the letters $\alpha, \beta, a, b$ is then said to be allowable if each two-letter subword $u_{i} u_{i+1}$ is among these eight allowable two-letter words. So a word $u$ is allowable if there is a path in the template starting at the branch line $u_{0}$ and then passing successively through branch lines $u_{1}, u_{2}, \ldots$.

We are interested in closed orbits, which in this notation correspond to periodic words, say

$$
u=w w w \ldots=w^{\infty}
$$

where $w=u_{0} u_{1} \ldots u_{n}$ is a finite word in our symbols. If this $u$ is allowable, we will then also say that the cyclic word $w$ is allowable. This notion of allowable cyclic words thus gives a way of describing orbits in our template.

However, given an allowable cyclic word, we need to know how pieces of the corresponding closed orbit fit together at each branch line in order for the entire orbit to embed in the template. Each orbit in fact has a unique correct ordering of its arcs along its branch lines. To determine this ordering, first choose one of the two possible orientations on the 
branch lines such that the flow from one to another preserves this order - say the one indicated in Figure 13. The following orderings are then immediately induced on allowable two-letter words:

$$
\alpha a<\alpha \beta, \quad \beta \alpha<\beta b, \quad b \beta<b a, \quad a b<a \alpha .
$$

This in turn induces the lexicographical ordering on all allowable words $u=u_{0} u_{1} u_{2} \ldots$ with a fixed first symbol $u_{0}$. The orbit corresponding to any allowable word will then embed in the template by ordering the pieces of orbit passing through any branch line $u_{0}$ according to the order of the corresponding words beginning with $u_{0}$. For a more detailed explanation of this symbolic dynamics description, see [Birman and Williams 1983; Ghrist et al. 1997].

\section{Obtaining Data Associated with Closed Orbits}

The method above of describing orbits allows the easy computation of various topological invariants. One of these is the period $p(u)$ of a closed orbit $u$ with respect to the fibration of the figure-eight knot complement. Since $p(u)$ is just the linking number of the closed orbit $u$ with the figure-eight knot $k$, we can compute it from Figure 13. There, the figureeight knot crosses over the template six times - at all strips except $\beta \alpha$ and $b a-$ and each crossing has the same sign. Hence we can write

$$
p\left(u_{i} u_{i+1}\right)= \begin{cases}0 & \text { if } u_{i} u_{i+1}=\beta \alpha \text { or } b a \\ 1 & \text { otherwise }\end{cases}
$$

and compute the overall period as a sum over the strips traversed. This also shows that the best possible lower and upper bounds on the length $l$ of a word whose orbit is of a given period $p$, are given by

$$
p \leq l \leq 2 p-2 \text {. }
$$

The cyclic word for a closed orbit also tells us which geodesic from Snap's list is in its homotopy class. To see this, first collapse the figure-eight template down to a directed graph with four vertices, corresponding to the branch lines, and eight edges, the strips between them. After ambient isotopy, this directed graph lies in the complement of our standard projection of the figure-eight knot $k$ as in Figure 14.

Now associate with each directed edge a word in SnapPea's generators of $\pi_{1}\left(S^{3}-k\right)$ via Figure 4 . There is some freedom in the choice of words here,

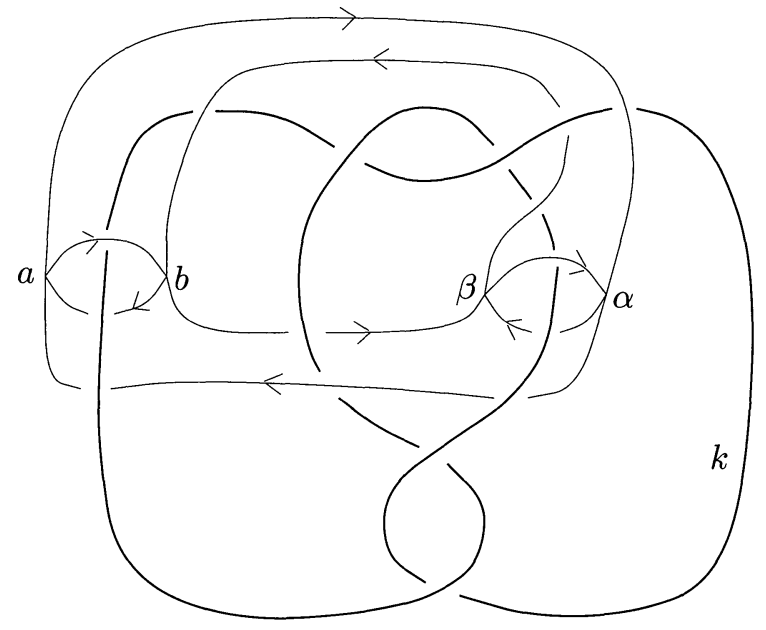

FIGURE 14. The figure-eight template can be collapsed to a directed graph in $S^{3}-k$.

depending on whether the vertices are taken to be above or below the projection of $k$, but since we are dealing only with closed orbits, any consistent choice will give the same result. One such labelling \{edges $\rightarrow \rightarrow \pi_{1}\left(S^{3}-k\right)$ is

$$
\begin{aligned}
& a b \mapsto 1, \quad b a \mapsto a, \quad \beta \alpha \mapsto b^{-1}, \quad \alpha \beta \mapsto c, \\
& a \alpha \mapsto 1, \quad b \beta \mapsto a, \quad \beta b \mapsto b^{-1} c, \quad \alpha a \mapsto c b^{-1} a .
\end{aligned}
$$

By taking the product of these words in SnapPea's generators over all strips traversed by a closed orbit, we obtain the free homotopy class of the orbit in $\pi_{1}\left(S^{3}-k\right)$, and Snap can then determine the corresponding geodesic.

Since each closed orbit in our flow can be expressed as a finite-length word in the letters $\alpha, \beta$, $a, b$, the problem of systematically generating pictures of all closed orbits has been reduced to the basic task of listing allowable words. A simple computer program can generate a list of all such words yielding distinct closed orbits on the template, up to a specified length. Using (4-1) we can choose this length such that all orbits up to a given period are included.

A secondary program can then turn words on this list directly into SnapPea link projection files for their corresponding closed orbits. Each of these files depicts in SnapPea a projection of a 2-component link with first component the figure-eight knot and second component the closed orbit in question, positioned according to the template on which it sits. An example is shown in Figure 15. 


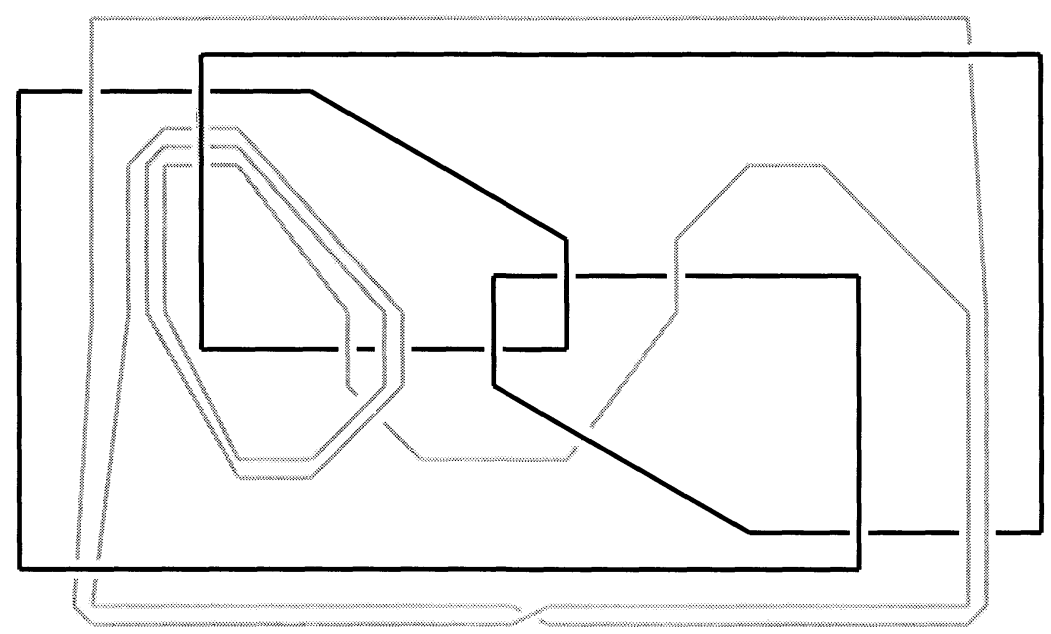

FIGURE 15. The link projection produced in SnapPea for the closed orbit given by the cyclic word $\alpha a \alpha a b a b a b \beta$.

With these SnapPea files for the closed orbits in the suspension flow on $S^{3}-k$, we can drill the orbits and obtain topological data about their complements.

\section{Analysis}

Using methods from Section 3, we find that all but four closed orbits of those up to period five are isotopic to their geodesic, the exceptions corresponding to the symmetry orbit of geodesics $\{257,259,267$, $268\}$. This is indicated in Table 4, which lists all the closed orbits up to period five, along with a range of associated data. These four nongeodesic closed orbits also provide further counterexamples to the suggestion of a geodesic having the least-volume complement in its homotopy class. For while the complements of the closed orbits have volume 8.107090, the geodesic complements have volume 10.962729 .

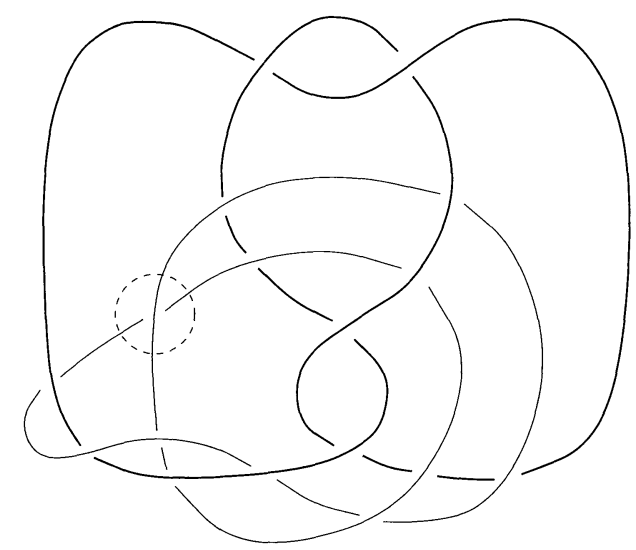

The special properties of these closed orbits suggest a more detailed study of them. Figure 16 shows pictures of closed orbit $\alpha a \alpha a b a$ and its corresponding geodesic, number 257, in the complement of our usual projection of the figure-eight knot.

We firstly note that a single crossing change in the circled region distinguishes the closed orbit from the geodesic. Moreover, this crossing change alters the curve's knot type in $S^{3}$ - the closed orbit is a trefoil knot when viewed in $S^{3}$, while the geodesic is a trivial knot. Table 4 also shows that while for the closed orbits listed, complement volume typically increases as the length of corresponding geodesic increases, the closed orbits in question have very low-volume complements for their geodesic length.

Their associated geodesics also have very small tube radii - over 15 times smaller than that for any other closed orbit up to period five. So relatively,

FIGURE 16. Homotopic curves in the figure-eight knot complement: on the left, the closed orbit $\alpha a \alpha a b a$ with complement volume 8.107090, and on the right the geodesic no. 257 with complement volume 10.962729 .

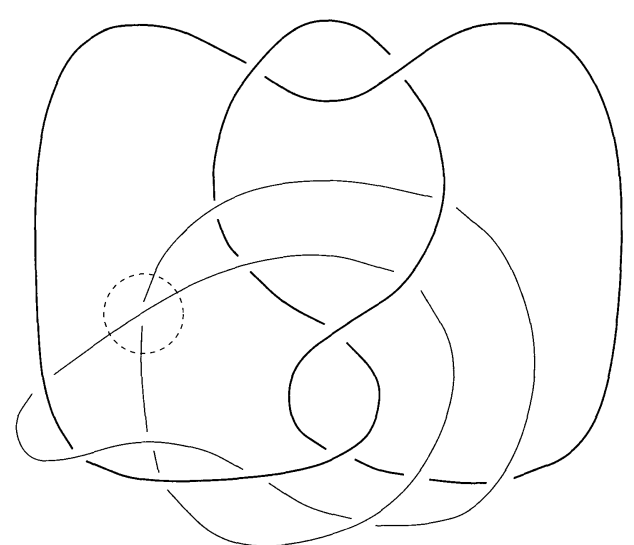




\begin{tabular}{|c|c|c|c|c|c|}
\hline cyclic word & period & $\begin{array}{l}\text { complement } \\
\text { volume }\end{array}$ & $\begin{array}{l}\text { geodesic } \\
\text { number }\end{array}$ & complex length & tube radius \\
\hline$\alpha a$ & 2 & 5.333490 & 6 & $1.725109-0.921839 i$ & 0.211824 \\
\hline$b \beta$ & 2 & 5.333490 & 7 & $1.725109+0.921839 i$ & 0.211824 \\
\hline$\alpha \beta \alpha a$ & 3 & 6.290303 & 13 & $2.416113-1.208686 i$ & 0.111840 \\
\hline$\alpha \beta b \beta$ & 3 & 6.290303 & 15 & $2.416113+1.208686 i$ & 0.111840 \\
\hline$\alpha \beta b a$ & 3 & 8.119533 & 16 & $2.633916+0.000000 i$ & 0.271768 \\
\hline$\alpha a b \beta$ & 3 & 8.119533 & 16 & $2.633916+0.000000 i$ & 0.271768 \\
\hline$\alpha a b a$ & 3 & 6.290303 & 12 & $2.416113-1.208686 i$ & 0.111840 \\
\hline$b \beta b a$ & 3 & 6.290303 & 14 & $2.416113+1.208686 i$ & 0.111840 \\
\hline$\alpha \beta \alpha \beta \alpha a$ & 4 & 6.770817 & 34 & $2.921563-1.381744 i$ & 0.071578 \\
\hline$\alpha \beta \alpha \beta b \beta$ & 4 & 6.770817 & 36 & $2.921563+1.381744 i$ & 0.071578 \\
\hline$\alpha \beta \alpha \beta b a$ & 4 & 9.340971 & 74 & $3.369922+0.334206 i$ & 0.117776 \\
\hline$\alpha \beta \alpha a b \beta$ & 4 & 9.340971 & 72 & $3.369922-0.334206 i$ & 0.117776 \\
\hline$\alpha \beta \alpha a b a$ & 4 & 8.519184 & 57 & $3.325772-1.498938 i$ & 0.105419 \\
\hline$\alpha \beta b a b \beta$ & 4 & 8.519184 & 63 & $3.325772+1.498938 i$ & 0.105419 \\
\hline$\alpha \beta b a b a$ & 4 & 9.340971 & 71 & $3.369922-0.334206 i$ & 0.117776 \\
\hline$\alpha a b a b \beta$ & 4 & 9.340971 & 73 & $3.369922+0.334206 i$ & 0.117776 \\
\hline$\alpha a b a b a$ & 4 & 6.770817 & 32 & $2.921563-1.381744 i$ & 0.071578 \\
\hline$b \beta b a b a$ & 4 & 6.770817 & 35 & $2.921563+1.381744 i$ & 0.071578 \\
\hline$\alpha \beta \alpha a \alpha a^{*}$ & 5 & 8.107090 & 259 & $4.174849-2.120825 i$ & 0.003249 \\
\hline$\alpha \beta b \beta \alpha a$ & 5 & 10.852301 & 244 & $4.126874+0.000000 i$ & 0.117758 \\
\hline$\alpha \beta b \beta b \beta^{*}$ & 5 & 8.107090 & 268 & $4.174849+2.120825 i$ & 0.003249 \\
\hline$\alpha \beta b \beta b a$ & 5 & 10.917658 & 332 & $4.312773+0.836995 i$ & 0.165149 \\
\hline$\alpha \beta b a \alpha a$ & 5 & 10.917658 & 324 & $4.312773-0.836995 i$ & 0.165149 \\
\hline$\alpha a \alpha a b \beta$ & 5 & 10.917658 & 325 & $4.312773-0.836995 i$ & 0.165149 \\
\hline$\alpha a \alpha a b a^{*}$ & 5 & 8.107090 & 257 & $4.174849-2.120825 i$ & 0.003249 \\
\hline$\alpha a b \beta b \beta$ & 5 & 10.917658 & 333 & $4.312773+0.836995 i$ & 0.165149 \\
\hline$\alpha a b \beta b a$ & 5 & 10.852301 & 238 & $4.126874+0.000000 i$ & 0.117758 \\
\hline$b \beta b \beta b a^{*}$ & 5 & 8.107090 & 265 & $4.174849+2.120825 i$ & 0.003249 \\
\hline$\alpha \beta \alpha \beta \alpha \beta \alpha a$ & 5 & 7.047485 & 66 & $3.325772-1.498938 i$ & 0.049991 \\
\hline$\alpha \beta \alpha \beta \alpha \beta b \beta$ & 5 & 7.047485 & 68 & $3.325772+1.498938 i$ & 0.049991 \\
\hline$\alpha \beta \alpha \beta \alpha \beta b a$ & 5 & 9.835917 & 176 & $3.916589+0.504028 i$ & 0.066257 \\
\hline$\alpha \beta \alpha \beta \alpha a b \beta$ & 5 & 9.835917 & 171 & $3.916589-0.504028 i$ & 0.066257 \\
\hline$\alpha \beta \alpha \beta \alpha a b a$ & 5 & 10.194877 & 192 & $3.953821-1.647569 i$ & 0.058246 \\
\hline$\alpha \beta \alpha \beta b a b \beta$ & 5 & 10.194877 & 201 & $3.953821+1.647569 i$ & 0.058246 \\
\hline$\alpha \beta \alpha \beta b a b a$ & 5 & 10.852301 & 243 & $4.126874+0.000000 i$ & 0.117758 \\
\hline$\alpha \beta \alpha a b a b \beta$ & 5 & 10.852301 & 239 & $4.126874+0.000000 i$ & 0.117758 \\
\hline$\alpha \beta \alpha a b a b a$ & 5 & 10.194877 & 188 & $3.953821-1.647569 i$ & 0.058246 \\
\hline$\alpha \beta b a b a b \beta$ & 5 & 10.194877 & 199 & $3.953821+1.647569 i$ & 0.058246 \\
\hline$\alpha \beta b a b a b a$ & 5 & 9.835917 & 174 & $3.916589-0.504028 i$ & 0.066257 \\
\hline$\alpha a b a b a b \beta$ & 5 & 9.835917 & 178 & $3.916589+0.504028 i$ & 0.066257 \\
\hline$\alpha a b a b a b a$ & 5 & 7.047485 & 61 & $3.325772-1.498938 i$ & 0.049991 \\
\hline$b \beta b a b a b a$ & 5 & 7.047485 & 67 & $3.325772+1.498938 i$ & 0.049991 \\
\hline
\end{tabular}

TABLE 4. Data associated with closed orbits (written as cyclic words from their template description) in the suspension flow of the figure-eight knot complement, and their corresponding geodesics. Orbits not isotopic to the geodesic in their homotopy class are marked with an asterisk. 
it takes only a small perturbation of these geodesics to change their isotopy class. This may help account for the fact that the closed orbits under the suspension flow, while satisfying Sakai's conditions and possessing an additional certain "straightness" property by their construction, are not necessarily geodesic.

\section{CONCLUSION}

After establishing in Section 2 that many hyperbolic three-manifolds contain (infinitely) many simple closed geodesics, we have endeavoured in this paper to understand some of the topology of the geodesics in the figure-eight knot complement.

In Section 3 we developed techniques for drawing explicit pictures of the geodesics in this manifold, by determining their correct isotopy class within a free homotopy class of closed curves. From here we observed that, while not true in general, many (short) geodesic knots seem to have the lowest-volume complement of all closed curves in their homotopy class. The additional observation of a certain 'simplicity' occuring in geodesics is somewhat vaguer and open to interpretation. It would be interesting to find more precise ways of expressing this quality.

In Section 4, we saw that despite several suggestive properties, not all closed orbits in the suspension flow of the monodromy for the figure-eight knot complement are geodesics. By studying the counterexamples uncovered, we could however make some valuable observations. In particular we saw that if a geodesic has small tube radius, it may be hard to distinguish topologically from other curves in its homotopy class, as even small perturbations can change its isotopy class. Hence it may be difficult to find topological conditions completely characterising geodesics. We could however still hope to find sufficient topological conditions for showing that a closed curve is a geodesic with relatively large tube radius.

While this paper has focussed on the case of the figure-eight knot complement, it would be most interesting to understand the topology of geodesics in general finite volume hyperbolic three-manifolds.

Some interesting questions for further investigation are:
Question 1. Are there infinitely many simple closed geodesics in every hyperbolic three-manifold of finite volume?

Question 2. In finite volume hyperbolic three-manifolds, are there topological conditions guaranteeing that in a homotopy class of closed curves, the geodesic is the one with the lowest-volume complement?

Question 3. In a hyperbolic three-manifold fibring over the circle, are there topological conditions guaranteeing that a closed orbit in its suspension flow is geodesic?

Question 4. In a cusped hyperbolic three-manifold $M$, do geodesics represent infinitely many knot types in each manifold obtained by Dehn filling on $M$ ?

Question 5. In a hyperbolic three-manifold fibring over the circle, does a closed orbit under the suspension flow have the lowest-volume complement of all curves in its homotopy class?

\section{ACKNOWLEDGEMENTS}

Thanks to Craig Hodgson, Walter Neumann and Oliver Goodman for their help.

\section{REFERENCES}

[Adams et al. 1999] C. Adams, J. Hass, and P. Scott, "Simple closed geodesics in hyperbolic 3-manifolds", Bull. London Math. Soc. 31:1 (1999), 81-86.

[Birman and Williams 1983] J. S. Birman and R. F. Williams, "Knotted periodic orbits in dynamical system, II: Knot holders for fibered knots", pp. 1-60 in Low-dimensional topology (San Francisco, 1981), edited by J. Samuel J. Lomonaco, Contemp. Math. 20, Amer. Math. Soc., Providence, RI, 1983.

[Bredon 1993] G. E. Bredon, Topology and geometry, Graduate Texts in Math. 139, Springer, New York, 1993.

[Brown 1971] R. F. Brown, The Lefschetz fixed point theorem, Scott, Foresman, Glenview, IL, 1971.

[Coulson et al. 2000] D. Coulson, O. A. Goodman, C. D. Hodgson, and W. D. Neumann, "Computing arithmetic invariants of 3-manifolds", Experiment. Math. 9:1 (2000), 127-152.

[Dowty 2000] J. Dowty, Ortholengths and hyperbolic Dehn surgery, Ph.D. thesis, University of Melbourne, 2000 . 
[Dubois 1998] J. Dubois, "Nœuds Fox-résiduellement nilpotents et rigidité virtuelle des variétés hyperboliques de dimension 3", Ann. Inst. Fourier (Grenoble) 48:2 (1998), 535-551.

[Francis 1987] G. K. Francis, A topological picturebook, Springer, New York, 1987.

[Ghrist et al. 1997] R. W. Ghrist, P. J. Holmes, and M. C. Sullivan, Knots and links in three-dimensional flows, Lecture Notes in Math. 1654, Springer, Berlin, 1997.

[Goodman et al. 1998] O. A. Goodman, C. D. Hodgson, and W. D. Neumann, "Snap", software, 1998. See http://www.ms.unimelb.edu.au/ snap. The Tube program by Goodman can also be found there.

[Hodgson and Weeks 1994] C. D. Hodgson and J. R. Weeks, "Symmetries, isometries and length spectra of closed hyperbolic three-manifolds", Experiment. Math. 3:4 (1994), 261-274.

[Jones and Reid 1997] K. N. Jones and A. W. Reid, "Geodesic intersections in arithmetic hyperbolic 3manifolds", Duke Math. J. 89:1 (1997), 75-86.

[Kojima 1988] S. Kojima, "Isometry transformations of hyperbolic 3-manifolds", Topology Appl. 29:3 (1988), 297-307.

[Miller $\geq 2001]$ S. M. Miller, Ph.D. thesis, University of Melbourne. In preparation.
[Morgan 1984] J. W. Morgan, "On Thurston's uniformization theorem for three-dimensional manifolds", pp. 37-125 in The Smith conjecture (New York, 1979), edited by J. W. Morgan and H. Bass, Pure appl. math. 112, Academic Press, Orlando, FL, 1984.

[Otal 1996] J.-P. Otal, Le théorème d'hyperbolisation pour les variétés fibrées de dimension 3, Astérisque 235, 1996.

[Phillips et al. 1993] M. Phillips, S. Levy, and T. Munzner, "Geomview: an interactive geometry viewer", Notices Amer. Math. Soc. 40 (October 1993), 985988. See http://www.geom.umn.edu/locate/geomview.

[Sakai 1991] T. Sakai, "Geodesic knots in a hyperbolic 3-manifold", Kobe J. Math. 8:1 (1991), 81-87.

[Thurston 1979] W. P. Thurston, "The geometry and topology of 3-manifolds", lecture notes, 1979. See www.msri.org/publications/books/gt3m.

[Thurston 1986] W. P. Thurston, "Hyperbolic structures on 3-manifolds, II: Surface groups and 3-manifolds which fiber over the circle", preprint, 1986. See http:// www.arxiv.org/abs/math.GT/9801045.

[Thurston 1997] W. P. Thurston, Three-dimensional geometry and topology, 1, Princeton Univ. Press, Princeton, NJ, 1997.

[Weeks 1993] J. R. Weeks, "SnapPea", software, 1993. Available at http://www.northnet.org/weeks/index/ SnapPea.html.

Sally M. Miller, Department of Mathematics and Statistics, University of Melbourne, Victoria 3010, Australia (S.Miller@ms.unimelb.edu.au)

Received December 20, 1999; accepted in revised form January 30, 2001 